\title{
Recent advances on fiber-based laser and Lidar systems for future space-borne monitoring of greenhouse gas
}

Nicolas Cézard, Agnès Dolfi-Bouteyre, Anne Durécu, Benoit Faure, Didier Goular, et al.

Nicolas Cézard, Agnès Dolfi-Bouteyre, Anne Durécu, Benoit Faure, Didier Goular, François Gustave, Philippe-Jean Hébert, Julien Lahyani, Julien Le Gouët, François Lemaître, Laurent Lombard, William Patiño, Christophe Planchat, Matthieu Valla, "Recent advances on fiber-based laser and Lidar systems for future space-borne monitoring of greenhouse gas," Proc. SPIE 11852, International Conference on Space Optics - ICSO 2020, 118521V (11 June 2021); doi: 10.1117/12.2599327

SPIE Event: International Conference on Space Optics - ICSO 2021, 2021, Online Only 


\section{International Conference on Space Optics-ICSO 2020}

Virtual Conference

30 March-2 April 2021

Edited by Bruno Cugny, Zoran Sodnik, and Nikos Karafolas
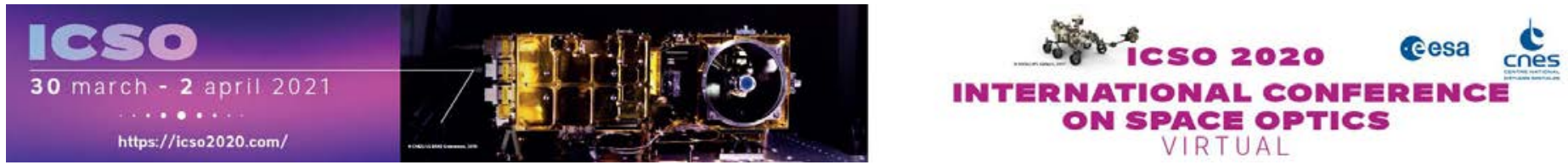

\section{Recent advances on fiber-based laser and Lidar systems for future space-borne monitoring of greenhouse gas}

\section{Cesa isoporecestings denes}




\title{
Recent advances on fiber-based laser and lidar systems for future space-borne monitoring of greenhouse gas
}

\author{
Nicolas Cézard*a ${ }^{*}$ Agnès Dolfi-Bouteyre ${ }^{\mathrm{b}}$, Anne Durécu ${ }^{\mathrm{b}}$, Benoit Faure ${ }^{\mathrm{c}}$, Didier Goular ${ }^{\mathrm{b}}$, François \\ Gustave $^{\mathrm{b}}$, Philippe-Jean Hébert ${ }^{\mathrm{c}}$, Julien Lahyani ${ }^{\mathrm{b}}$, Julien Le Gouët ${ }^{\mathrm{b}}$, François Lemaître ${ }^{\mathrm{a}}$, Laurent \\ Lombard $^{\mathrm{b}}$, William Patiño ${ }^{\mathrm{a}}$, Christophe Planchat ${ }^{\mathrm{b}}$, Matthieu Valla ${ }^{\mathrm{b}}$ \\ ${ }^{a}$ ONERA/DOTA, Université de Toulouse, F-31055 Toulouse France \\ ${ }^{b}$ DOTA, ONERA, Université Paris Saclay, F-91123 Palaiseau, France \\ ${ }^{\mathrm{c}} \mathrm{CNES}, 18$ av. E. Belin, 31401 Toulouse, France
}

\begin{abstract}
We report on recent advances at ONERA on fiber-based laser and lidar systems, in the perspective of future space-borne monitoring of greenhouse gas, especially $\mathrm{CO}_{2}$ and $\mathrm{CH}_{4}$. We first present an advancement status of research activities on high-peak-power single-frequency fiber lasers at $2.05 \mu \mathrm{m}$ and $1.58 \mu \mathrm{m}$ for $\mathrm{CO}_{2}$ monitoring, and at $1.64 \mu \mathrm{m}$ for $\mathrm{CH}_{4}$, as well as perspectives for future amplification and frequency-stabilization of these lasers. The second parts deals with activities led on electro-optic dual comb spectroscopy (EO-DCS), which could be a potential future alternative technique to conventional space-borne IPDA lidars (Integrated Path Differential Absorption). We report on an EO-DCS experiment performed in pulsed regime, using an Er-Yb-doped amplifier delivering up to $92 \mu \mathrm{J}, 2 \mu$ s pulses at $10 \mathrm{kHz}$ repetition rate. An absorption line of $\mathrm{HCN}$ is sampled simultaneously by a 13-teeth frequency comb, and its transmission is measured with a good agreement with respect to the expectation.
\end{abstract}

Keywords: Fiber laser, greenhouse gas, frequency comb, spectroscopy

\section{INTRODUCTION}

Monitoring the concentration of greenhouse gas (GHG) in the atmosphere from a space-borne observatory, is a key for better localization of GHG sources, sinks, and fluxes at the global scale, as well as better understanding of their impact on climate evolution. Complementary to passive infrared observation, lidar monitoring of GHG from space could offer good spatial coverage at high latitudes, night and day and four-seasons data availability, and low sensitivity to bias sources such as aerosols layers or complex surfaces scattering [1]. Lidar technologies for space-borne observation of GHG have been considered since at least two decades [2]. For $\mathrm{CO}_{2}$, the European A-SCOPE study and the NASA ASCENDS program have permitted to derive observational objectives and technical requirements for space-borne lidars, and also to develop many technological approaches to fulfill them. Two main spectral windows have been especially selected: the $2.05 \mu \mathrm{m}$ region and the $1.57-1.58 \mu \mathrm{m}$ region. The former offers a higher weighting function in the troposphere, thus allowing relaxing some technical requirements compared to the latter, but conversely, the 1.57$1.58 \mu \mathrm{m}$ spectral window is more attractive in terms of detection sensitivity and diversity of market-available highquality optronic components. For $\mathrm{CH}_{4}$, the European MERLIN project has selected the $1.65 \mu \mathrm{m}$ spectral window as the most appropriate for methane observation from space. Based on a solid-state laser source (Optical Parametric Oscillator or OPO), the MERLIN lidar should be put in orbit in a few years $(\sim 2025)$, and will be the first space-borne lidar dedicated to a GHG (methane) [1] .

In general, solid-state laser sources based on crystal cavities are very efficient to deliver high peak-power pulses (many $\mathrm{kW}$ ), and therefore they represent one of the most promising approach for future space-borne lidars. However, they also involve large numbers of free-space optics, which can raise substantial alignment issues, especially when vibrating environments are considered. On the contrary, fiber-based lasers (and lidars) allow carrying light from a component to 
another without any alignment problem. Consequently, fiber-based systems are intrinsically robust solutions, very attractive for space-borne applications. However, in standard optical fibers, non-linear effects, especially Stimulated Brillouin Scattering (SBS), limit the extractible peak-power, in the range of 10-100 W typically (depending of the fiber length). ONERA has been investigating techniques to overcome Brillouin limits for more than a decade. Implementing various techniques to mitigate SBS and deliver high peak-power single-frequency pulses, ONERA's laboratory has developed up to now three fiber-based laser systems at wavelengths that coincide with strong absorption lines of $\mathrm{CO}_{2}$ and $\mathrm{CH}_{4}$. In the first part of this paper, we present an advancement status on these laser sources as well as perspectives for their future amplification and frequency-stabilization.

Fiber technology is also very attractive in that it offers very convenient means to apply intensity and phase modulation patterns to the optical signal. This opens original opportunities for spectroscopic lidar measurements, which could eventually lead, in the future, to new concepts for space-borne GHG sounding systems. Electro-Optic Dual Comb Spectroscopy is one of these new concepts under assessment by ONERA and CNES. The principle was presented in details during the last ICSO conference three years ago [3], and demonstrated in continuous mode and at low power. In the second part of this paper, EO-DCS principle is briefly recalled and we report on an absorption spectroscopy experiment (in an HCN gas cell) where a dual-comb is pulsed and amplified up to $92 \mu \mathrm{J}, 2 \mu \mathrm{s}, 10 \mathrm{kHz}$ pulses in an ErYb-doped fiber amplifier.

\section{MOPA FIBER LASER SOURCES FOR GHG-MONITORING LIDARS}

\subsection{Current advancement status}

The fiber laser sources developed at ONERA generally follow a MOPA fiber architecture (Master Oscillator Power amplifier), schemed in Figure 1. The Master Oscillator (MO) is typically a continuous-wave, low-power, linearly polarized, spectrally-narrow laser emitter at the appropriate wavelength (most of the time a Distributed Feedback Diode or DFB). It is followed by an intensity modulator (IM) for temporal pulse-shaping. It can be for instance a MachZehnder Modulator (MZM), a binary optical switch, or an Acousto-Optic Modulator (AOM). In coherent lidar architectures, the AOM is often the preferred solution, since it can provide at the same time a slight frequency shift (tens of $\mathrm{MHz}$ ) that serves as an intermediate frequency for heterodyne lidar measurements. The IM output delivers low-energy laser pulses with the desired temporal profile, tailored so as to pre-compensate the pulse distortion induced by the subsequent amplifier stages. The Power Amplifier (PA) is often divided in several stages, in order to distribute the gain over different pieces of fiber, optimize fiber properties (length, diameter...) as the pulse energy is growing, and prevent excessive heat and noise accumulation. As the peak power is growing, Stimulated Brillouin Scattering (SBS) becomes a limiting process, and SBS-mitigation techniques must be employed to circumvent this limit. Working on special fibers (glass composition, large mode-field diameters, distributed mechanical strains...), ONERA has reported state-of-the-art high-peak power single-frequency fiber-based laser sources [4][5]. Table 1 summarizes the performances of three fiber MOPA sources built especially for GHG monitoring.

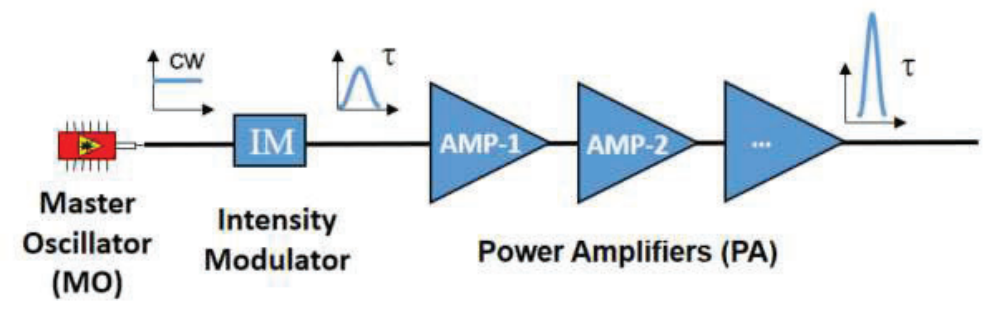

Figure 1 - General scheme of a fiber Master Oscillator Power Amplifier (MOPA) architecture

The $1.58 \mu \mathrm{m}$ HEPILAS fiber laser was demonstrated in 2014 under specifications from the European Space Agency (ESA), and features a three-stage Er-doped power amplifier [6]. Though the $2 \mathrm{~mJ}$-objective for the ON-line wavelength could not be met during the study, the laser exceeded the $0.2 \mathrm{~mJ}$-objective for the OFF-line wavelength, which was a very encouraging step. 
The $2.05 \mu \mathrm{m}$ HELENA laser source features a four-stage Tm-doped MOPA architecture. Based on a former laser source [7], it has been very recently upgraded and optimized in the perspective of $\mathrm{CO}_{2}$ lidar monitoring [8]. The source has been integrated into a coherent lidar architecture, and first $\mathrm{CO}_{2}$ measurements have just been realized (reported in [9]). In both HELENA and HEPILAS lasers, Large Mode Area (LMA) fibers and distributed mechanical strains have been used efficiently as SBS-mitigation techniques.

The $1.64 \mu \mathrm{m}$ VEGA fiber laser, completed in 2018, has been built for methane leaks monitoring from a ground-based lidar system, in partnership between ONERA and TOTAL. Since there are no efficient doping ions at $1.64 \mu \mathrm{m}$, the VEGA laser features a two-stage Raman amplifier [10]. In that case, the fiber core diameter must remain very narrow to enhance Raman non-linearity, and using LMA fibers was not an option. Therefore, only the distributed strain technique has been applied for SBS-mitigation at the signal wavelength. Moreover, since Raman amplifiers require high peakpower pumping lasers, SBS-mitigation also had to be implemented for pump lasers (using frequency chirps techniques). VEGA has been operated successfully with coherent detection to monitor industrial methane leaks in range-resolved mode, and it has also been tested in IPDA mode (Integrated Path Differential Absorption) on a hard target located 2.4 km away [11].

Table 1. Fiber-based laser sources developed at ONERA.

\begin{tabular}{|c|c|c|c|}
\hline Gas species & \multicolumn{2}{|c|}{$\mathrm{CO}_{2}$} & \multirow{2}{*}{$\begin{array}{c}\mathrm{CH}_{4} \\
\text { VEGA }\end{array}$} \\
\hline System Name & HELENA & HEPILAS & \\
\hline Wavelength & $2.05 \mu \mathrm{m}$ & $1.58 \mu \mathrm{m}$ & $1.64 \mu \mathrm{m}$ \\
\hline Pulse energy $(\boldsymbol{\mu} \boldsymbol{J})$ & 120 & 260 & 14 \\
\hline Pulse length (ns) & 200 & 150 & 100 \\
\hline Peak power $(\mathrm{W})$ & 600 & 1700 & 140 \\
\hline Pulse Rep. Rate (kHz) & 20 & 4 & 20 \\
\hline Average power (W) & 2.4 & 1 & 0.3 \\
\hline Active fiber type & Tm-doped fiber & Er-doped Fiber & Raman enhanced fiber \\
\hline Amplification stages & 4 & 3 & 2 \\
\hline $\begin{array}{c}\text { Brillouin mitigation } \\
\text { techniques }\end{array}$ & $\begin{array}{l}\text { Large Mode Area, } \\
\text { Distributed strain }\end{array}$ & $\begin{array}{l}\text { Large Mode Area, } \\
\text { Distributed strain }\end{array}$ & $\begin{array}{l}\text { Distributed strain (signal) } \\
\text { Frequency Chirp (pump) }\end{array}$ \\
\hline Beam Quality $\mathbf{M}^{2}$ & $<1.12$ & $<1.20$ & 1 (single-mode fiber) \\
\hline Reference & {$[8]$} & {$[6]$} & {$[10]$} \\
\hline
\end{tabular}

\subsection{Going further in amplification}

In their current states, these fiber-based laser sources already open very attractive perspectives to build innovative and robust ground based lidars dedicated to GHG monitoring at local scale (gas leak detection on industrial sites, urban areas GHG monitoring...). Our current efforts on that way especially go to HELENA and VEGA lidar systems $\left(\mathrm{CO}_{2}\right.$ at 2.05 $\mu \mathrm{m}$ and $\mathrm{CH}_{4}$ at $1.64 \mu \mathrm{m}$ ). But of course further amplification will be needed to fulfill requirements for future space-borne lidars. Several ways are currently considered at ONERA to amplify further our fiber-based laser sources (submitted patents). At $2.05 \mu \mathrm{m}$, the HELENA project is especially promising. After having developed the source reported in Table 1, the goal now is to amplify the output in a Ho:YLF crystal. This experiment is currently led by ONERA and the Laboratoire de Météorologie Dynamique (LMD). It is briefly described in [9]. The expected crystal gain depends of various parameters (repetition frequency, crystal length, beam focus...) but simulations indicate that several mJ could be obtained with a repetition rate higher than $1 \mathrm{kHz}(5-10 \mathrm{~W}$ average power). The general idea is to combine the benefits of fiber technology (settings versatility, pulse control, no alignments...) and crystal technology (very high peak power) into a single hybrid architecture (hence the name HELENA - Hybrid Laser Emitter for Environmental Applications), while minimizing the laser complexity in terms of free-space optics alignments (no free space cavity). This hybrid combination could be a breakthrough for future airborne and space-borne applications. 


\subsection{Frequency stabilization}

In classical IPDA lidar concepts for space-borne GHG monitoring, the emitted frequencies (especially the ON-line frequency) must be stabilized in order to avoid uncontrolled frequency drifts, which would convert into measurement bias on the gas concentration. For $\mathrm{CO}_{2}$, the absolute stability requirement is in the range of $100-200 \mathrm{kHz}(\mathrm{Allan}$ deviation) in $10 \mathrm{~s}$, while it is relaxed down to a few $\mathrm{MHz}$ for $\mathrm{CH}_{4}$ (indeed, for $\mathrm{CH}_{4}$ at $1.645 \mu \mathrm{m}$, the absorption crosssection at the ON-line wavelength is located in a trough with low frequency sensitivity). MOPA architectures are particularly convenient to install frequency-stabilization loops directly at the output of the Master Oscillator, since power amplifiers usually do not add any frequency jitter. We have designed a stabilization loop based on the Frequency Modulation Spectroscopy (FMS) technique [12]. Schemed in Figure 2, this technique is particularly efficient for highprecision frequency locking at the center of an absorption line in a gas cell. The demodulation signal (error signal for locking) looks like the absorption first derivative, and presents a maximal slope at the line center (the slope also depends of the lock-in phase setting, but the latter can be adjusted). Figure 3 shows the build I/Q demodulation circuit (radiofrequency components), as well as the demodulation signals that can be observed in channels $I, Q$, and $Z=\left(I^{2}+Q^{2}\right)^{1 / 2}$ (phase-independent). These experimental signals, obtained during tests conducted with a $\mathrm{C}_{2} \mathrm{H}_{2}$ gas cell at $1539 \mathrm{~nm}$ are in excellent agreement with expectations. We are currently implementing this technique to stabilize the VEGA lidar at $1645 \mathrm{~nm}$, using a gas cell filled with $\mathrm{CH}_{4}$. Work is ongoing to assess the obtained frequency stability (usually, this requires duplicating the setup and measuring the Allan deviation of the beat note between two stabilized lasers).

The FMS technique uses an external modulation mean, such as an electro-optic phase modulator (EOM), and is efficient for locking a laser at the center of an absorption line (it can also be slightly tuned off-center but the stability decreases since the error locking signal slope decreases). However, for space-borne observation of $\mathrm{CO}_{2}$, especially at $2.05 \mu \mathrm{m}$, the On-line frequency would be ideally quite far aside from the line center (typ. $2 \mathrm{GHz}$ ), so that the measurement weighting function in the troposphere (where sources and sinks are located) could be favored. In that case, the scheme of Figure 2 can still be used to lock a master oscillator on the line center of a gas cell, and a slave-oscillator can then be locked on the master oscillator in the radiofrequency domain (note that the slave-oscillator is the main oscillator of the MOPA architecture in that case). Here again the fiber technology is an asset for realizing such master-slave locking circuits. Eventually, the fiber technology can also be used to replace the gas cell by itself. For instance, using a hollow-core gasfilled Kagome fiber, Curtis et al. [13] have reported very compact architectures and excellent stability results at $2.05 \mu \mathrm{m}$ using the FMS locking technique.
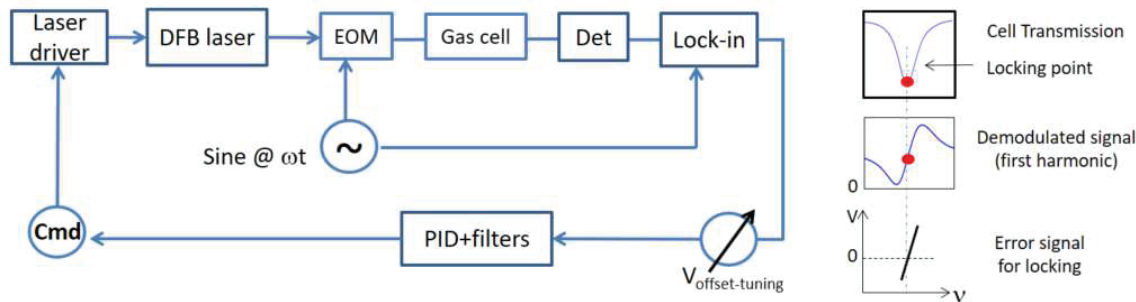

Figure 2 - Scheme of the frequency-modulation technique used to lock a DFB laser at the center of an absorption line in a gas cell.
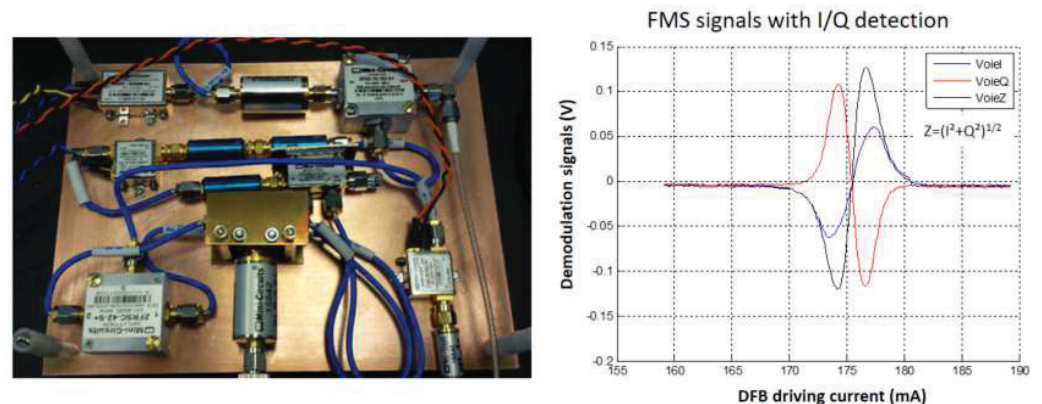

Figure 3 - Left: I/Q demodulation stage of the FMS-based stabilization loop. Right: Locking signals observed on channels I, Q, and Z. 


\section{ELECTRO-OPTIC DUAL-COMB SPECTROSCOPY LIDAR}

Besides developing high-peak power fiber/hybrid laser sources and frequency-stabilization sub-systems, ONERA also leads activities that look beyond the traditional double-wavelength IPDA technique (ON/OFF), and tries to revisit the gas measurement concept by itself, still using fiber technology. During the last ICSO conference in 2018, the concept of Electro-Optics Dual-Comb Spectroscopy (EO-DCS), as well as the SCALE mission principle (Short-Comb Atmospheric Lidar Experiment) have been presented in details by ONERA and CNES [3]. The paper also reported a feasibility demonstration by ONERA of EO-DCS in continuous-wave mode, and at low power. Here we report on an experiment where the short dual-comb is pulsed and amplified up to significant pulse energy $(92 \mu \mathrm{J})$. We briefly recall EO-DCS principle before, for the paper completeness.

\subsection{Principle and previous results}

The general idea of EO-DCS is to probe an atmospheric absorption line simultaneously at several frequency points, distributed along the absorption line, following a regular and stable short-comb pattern (between 5 and 15 teeth typically). Compared to the traditional and sequential two-frequency pattern (ON-OFF) involved in current IPDA spaceborne concepts (such as MERLIN), the measurement simultaneity at several frequencies would offer significant advantages. It would ensure that all the probed frequency points travel through exactly the same atmospheric column (cancelling any bias induced by the time lag between sequential On-Off patterns) and it would also relax the frequency stability and knowledge requirement (discussed in the precedent section). Indeed, a multi-frequency measurement should permit to retrieve continuously not only the atmospheric gas column-content, but also the frequency center of the absorption line (thus reducing bias induced by an inaccurate knowledge of its position). Potentially this could avoid adding a sophisticated frequency-stabilization sub-system in a future frequency-comb based space-borne lidar.

Short frequency combs can be generated in fiber technology using electro-optic phase modulators [14]. It is known that applying a radiofrequency (RF) sine modulation in an EOM converts a single-frequency input into a Bessel frequencycomb output. In [3], it has also been mentioned that by applying a RF frequency plus its second and third harmonics with convenient power levels, it is possible to generate a 9-teeth or even 13-teeth flat frequency-comb. Finally, it has also been presented that probing an absorption line with two distinct frequency combs with slightly different frequency steps and frequency offsets allows transferring the absorption line measurement from the optical domain to the RF domain [15][16]. This is the basis of what we refer as EO-DCS in this paper, schemed in Figure 4.
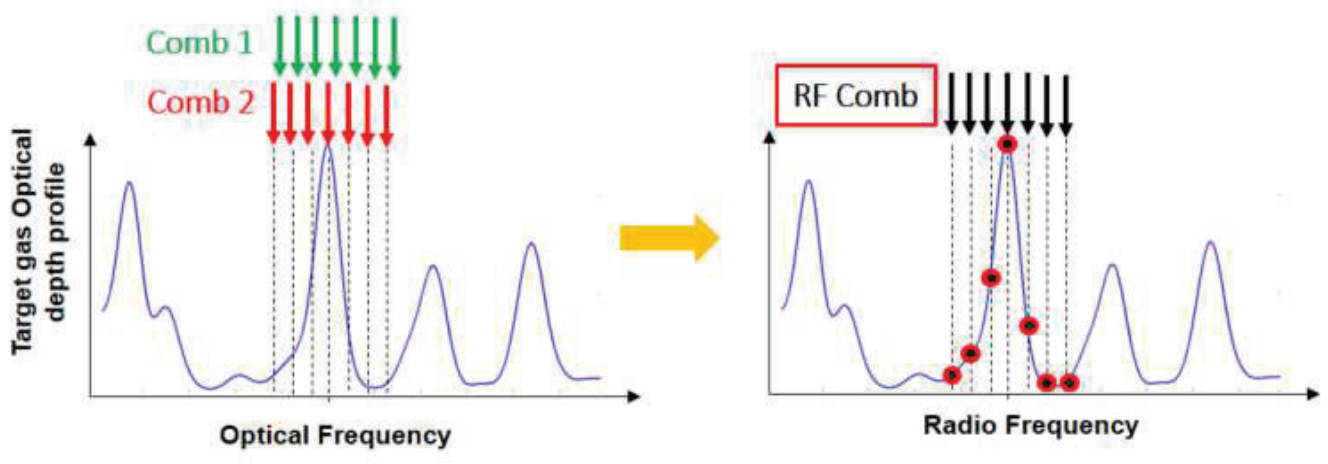

Figure 4 - Concept of simultaneous sampling of an absorption line in the RF domain using EO-DCS

\subsection{Amplified and pulsed EO-DCS}

The results reported in [3] have been obtained in the continuous-wave regime and with relatively low power $(<0.4 \mathrm{~W})$. However in the perspective of a space-borne system, it is necessary to evaluate EO-DCS principle in pulsed mode, and with significant pulse energy. Therefore, we have made an experiment, schemed in Figure 5, where the double-comb is amplified into two EDFAs (Erbium-Doped Fiber Amplifiers). The first one operates in cw-mode (same as in [3]), and the 
second operates in pulsed regime. The pulse shape is adjusted by an AOM located between the EDFAs (it also brings a $+40 \mathrm{MHz}$ shift). The pulse profile generated by the AOM is calculated so as to pre-compensate the pulse distortion that occurs in the pulsed EDFA. The output pulses are square-shaped or smooth-square-shaped, with a duration of $2 \mu \mathrm{s}$. The pulsed EDFA delivers a maximum power of $100 \mu \mathrm{J}$ at $10 \mathrm{kHz}$. It was operated here up to $92 \mu \mathrm{J}$. The frequency-combs are 13-teeth long. Their respective modulation frequencies are $\mathrm{f}_{\mathrm{MEO} 1}=497 \mathrm{MHZ}$ and $\mathrm{f}_{\mathrm{MEO} 2}=497.5 \mathrm{MHz}\left(\Delta \mathrm{f}_{\mathrm{MEO}}=500 \mathrm{kHz}\right)$, and the total offset frequency is $\mathrm{f}_{\mathrm{c}}=25.5 \mathrm{MHz}\left(=\mathrm{f}_{\mathrm{AOM} 3}-\left(\mathrm{f}_{\mathrm{AOM} 1}-\mathrm{f}_{\mathrm{AOM} 2}\right)\right)$. The optical wavelength is set at the middle of an $\mathrm{HCN}$ absorption line at $\lambda_{\mathrm{c}}=1545.23 \mathrm{~nm}$, noted $v_{\mathrm{c}}$ in frequency. Consequently, the probe comb is located at $v_{\mathrm{k}}=v_{\mathrm{c}}+\mathrm{kf} \mathrm{MEO}_{\mathrm{ME}}$, $\mathrm{k}=\{-6,+6\}$ in the optical domain, with a $6 \mathrm{GHz}$ total span, and at $\mathrm{f}_{\mathrm{k}}=\mathrm{f}_{\mathrm{c}}+\mathrm{k} \Delta \mathrm{f}_{\mathrm{MEO}}$ in the RF domain, with only a $6 \mathrm{MHz}$ span. The signal can thus be detected and digitized with a very limited bandwidth.

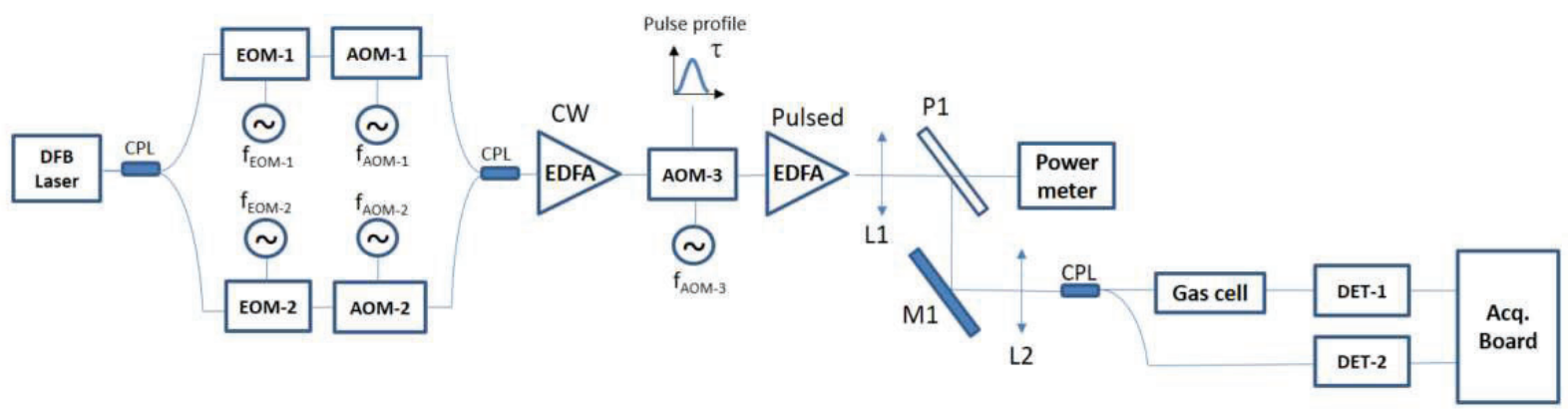

Figure 5 - Pulsed and amplified EO-DCS experiment scheme. (DFB: Distributed Feedback Laser, EOM: Electro-optic modulator, AOM: Acousto-optic modulator, CPL: fiber coupler, EDFA: Erbium-Doped Fiber Amplifier, P: glass plate, M: mirror, L: lens, DET: Detector)

Figure 6 (left) shows the time profile of an output pulse, after amplification. The interference pattern of the two combs is clearly visible. The $2 \mu$ s duration is chosen accordingly with the $500 \mathrm{kHz}$ frequency step of the comb in the RF domain. This choice ensures that the first zero of the sinc function associated with the square pulse profile coincides with the adjacent comb tooth. In that way, each tooth is efficiently isolated from its neighbor. We have measured the power associated with each tooth at the pulsed amplifier output, compared to the input teeth power distribution. It appeared that the pulsed EDFA gain is relatively homogeneous inside the comb, within $2 \mathrm{~dB}$ from the $1^{\text {st }}$ tooth to the $13^{\text {th }}$. The Figure 6 (right) shows the computed transmission profile of the HCN gas cell using the EO-DCS technique in pulsed mode at $76 \mu \mathrm{J}$. It is computed by integrating the power of each tooth in the frequency domain, and by performing the ratio between the power measured in the reference channel and in the gas cell channel (DET-2/DET-1 in Figure 5). Because the detector was slightly saturated here, the repeatability was not at its best for this measurement, but still the measured absorption line agrees very well with the expectation. It is thus demonstrated that EO-DCS is feasible in pulsed mode with significant pulse energy. In 2021, a similar experiment has been led with $500 \mu \mathrm{J}$ pulses, using a "home-made" highpeak power amplifier from ONERA, and will be reported soon. 

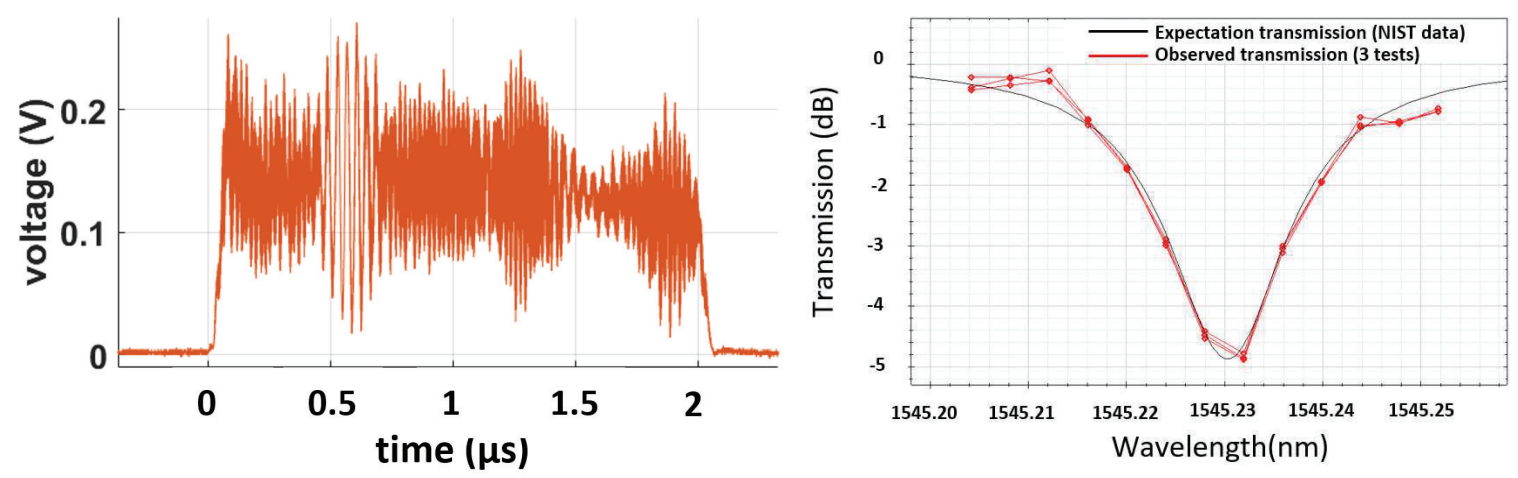

Figure 6 - Left: Time profile of a double-comb emitted pulse; Right: Measured transmission of the HCN line at $1545.23 \mathrm{~nm}$ in pulsed mode with $76 \mu \mathrm{J}, 10 \mathrm{kHz}, 2 \mu$ s square-shaped pulses.

\section{CONCLUSION}

We have presented three high-peak-power single-frequency laser sources developed at ONERA at $1.58 \mu \mathrm{m}, 2.05 \mu \mathrm{m}$ and $1.64 \mu \mathrm{m}$, and designed for greenhouse gas monitoring $\left(\mathrm{CO}_{2}\right.$ and $\left.\mathrm{CH}_{4}\right)$. Two of them have already been successfully integrated into lidar systems that have demonstrated their capability to effectively monitor the target gas. For ground-based systems, these results open very attractive perspectives for future industrial or urban applications. For space-borne applications, further amplification will be required. At $2.05 \mu \mathrm{m}$, a hybrid fiber/crystal architecture is currently under test with Ho:YLF crystals, with the objective to join the "best of both worlds", i.e. the versatility and robustness of the fiber technology, with the high-peak power of the crystal technology, without sacrificing much in free-space optics complexity. Similar approaches could also be tested at other wavelengths, with appropriate crystals. Frequency-stabilization is also an issue for conventional IPDA space-borne concepts. We have presented the FMS-based stabilization approach at ONERA. Though classical, this approach can be implemented very efficiently on fiber-based systems, including using all-fiber gas cells. Finally, we also explore alternative concepts to the conventional space-borne IPDA technique, taking advantage of the fiber technology versatility in terms of phase and intensity modulation techniques. The EO-DCS technique is especially attractive, in that it has the potential to measure not only the gas column content but also the emitted frequency (potentially relaxing stabilization system requirements). ONERA has demonstrated that pulsed EO-DCS is feasible with significant pulse energies $(92 \mu \mathrm{J}$ ), and further amplification (up to $500 \mu \mathrm{J}$ ) is currently under test. Various research activities are on their way at ONERA and CNES to continue exploring and assessing whether this technique will be applicable in future space-borne lidars.

\section{ACKNOWLEDGMENTS AND FUNDINGS}

The work presented here has been realized at ONERA's laboratories, with financial support from European Space Agency for the HEPILAS laser source (ESA contract \#4000104022/11/NL/CP), from Total for the VEGA lidar (NAOMI contract), and from CNES for the $2 \mu \mathrm{m}$ laser source upgrade (convention 5100018351; contract \#190192) and for the short-comb amplification experiment (contract \#R-S18/OT-0002-094).

\section{REFERENCES}

[1] G. Ehret, P. Bousquet, C. Pierangelo, M. Alpers, B. Millet, J.B. Abshire, H. Bovensmann, J.P. Burrows, F. Chevallier, P. Ciais, C. Crevoisier, A.Fix, P. Flamant, C. Frankenberg, F. Gibert, B. Heim, M. Heimann, S. Houweling, H.W. Hubberten, P. Jöckel, K. Law, A. Löw, J. Marshall, A. Agusti-Panareda, S. Payan, C. 
Prigent, P. Rairoux, T. Sachs, M. Scholze, M. Wirth, "MERLIN: A French-German Space Lidar Mission Dedicated to Atmospheric Methane," Remote Sens. 2017, 9, 1052.

[2] Singh, U. N., Walsh, B. M., Yu, J., Petros, M., Kavaya, M. J., Refaat, T. F. and Barnes, N. P., "Twenty years of Tm:Ho:YLF and LuLiF laser development for global wind and carbon dioxide active remote sensing," Opt. Mater. Express, OME 5(4), 827-837 (2015).

[3] P. Hébert, F. Lemaître, "SCALE: validations and prospects for a novel type of sounding lidar using short frequency combs", in Proc. of International Conference on Space Optics (ICSO), (2018)

[4] G. Canat, S. Jetschke, S. Unger, L. Lombard, P. Bourdon, J. Kirchhof, V. Jolivet, A. Dolfi, and O. Vasseur, "Multifilament-core fibers for high energy pulse amplification at $1.5 \mu \mathrm{m}$ with excellent beam quality," Opt. Lett. 33, 2701-2703 (2008)

[5] F. Prevost, L. Lombard, J. Primot, L. P. Ramirez, L. Bigot, G. Bouwmans, and M. Hanna, "Coherent beam combining of a narrow-linewidth long-pulse Er3+-doped multicore fiber amplifier," Opt. Express 25, 95289534 (2017)

[6] G. Canat, J. Le Gouët, L. Lombard, A. Bresson, D. Goular, A. Dolfi-Bouteyre, S. Duzellier, D. Boivin, J. Nilsson, J. Sahu, S. Bordais, "High power pulsed fiber laser development for $\mathrm{CO}_{2}$ space based DIAL system", in Proc. of International Conference on Space Optics (ICSO), (2014)

[7] Lucas E., L. Lombard, Y. Jaouën, S. Bordais, and G. Canat, "1 kW peak power, 110 ns single-frequency thulium doped fiber amplifier at $2050 \mathrm{~nm}, "$ Appl. Opt. 53, 4413-4419 (2014)

[8] J. Lahyani, J. Le Gouët, F. Gibert, and N. Cézard, "2.05- $\mu \mathrm{m}$ all-fiber laser source designed for CO2 and wind coherent lidar measurements", accepted in Applied Optics (2021)

[9] J. Lahyani, N. Cézard, B. Faure, F. Gibert, J. Le Gouët, « 2- $\mu \mathrm{m}$ hybrid laser emitter for future carbon dioxyde space-borne lidar measurement », in Proc. of International Conference on Space Optics (ICSO), (2021)

[10]P. Benoit, S. Le Méhauté, J. Le Gouët, and G. Canat, "All-fiber laser source at $1645 \mathrm{~nm}$ for lidar measurement of methane concentration and wind velocity," Opt. Lett. 46, 126-129 (2021)

[11] N. Cézard, S. Le Méhauté, J. Le Gouët, M. Valla, D. Goular, D. Fleury, C. Planchat and A. Dolfi-Bouteyre, "Performance assessment of a coherent DIAL-Doppler fiber lidar at $1645 \mathrm{~nm}$ for remote sensing of methane and wind," Opt. Expr. 28, no. 15, pp. 22345-22357 (2020).

[12] G. C. Bjorklund, "Frequency-modulation spectroscopy: a new method for measuring weak absorptions and dispersions," Opt. Lett. 5, 15-17 (1980)

[13] E. Anne Curtis, Thomas Bradley, Geoffrey P. Barwood, Christopher S. Edwards, Natalie V. Wheeler, Richard Phelan, David J. Richardson, Marco N. Petrovich, and Patrick Gill, "Laser frequency stabilization and spectroscopy at $2051 \mathrm{~nm}$ using a compact CO2-filled Kagome hollow core fiber gas-cell system," Opt. Express 26, 28621-28633 (2018)

[14] A. Parriaux, K. Hammani, and G. Millot, "Electro-optic frequency combs," Adv. Opt. Photon. 12, 223-287 (2020)

[15]D. A. Long, A. J. Fleisher, K. O. Douglass, S. E. Maxwell, K. Bielska, J. T. Hodges, and D. F. Plusquellic, "Multiheterodyne spectroscopy with optical frequency combs generated from a continuous-wave laser", Opt. Lett. 39, 2688 (2014).

[16] P. Martín-Mateos, B. Jerez, and P. Acedo, "Dual electro-optic optical frequency combs for multiheterodyne molecular dispersion spectroscopy," Opt. Express 23, 21149-21158 (2015). 